\title{
Simulation and Optimization of Microcantilever Biosensors
}

\section{Linxia Gu*}

Department of Mechanical Engineering, University of Nebraska-Lincoln, USA

Biosensors, which convert biology excitation into measurable signals, are gaining interests across different research areas including biology, chemistry, electrical \& electronic engineering, and mechanical engineering. A typical biosensor usually consists of three parts: a biological recognition element, a transducer, and a detector element [1]. As one of the most common type of biosensors, microcantilever biosensor has been used to detect prostate cancer by identifying prostate-specific antigen [2], humidity [3], herbicide [4], metal ion [5], temperature and radiation [6] by applying specific biological recognition element on the cantilever surface. The development of microcantilever biosensors has been summarized by Hwang et al. [7]. When the biological recognition element was stimulated by biomolecular surface stress of cantilever, it led to deflection. The relationship between the stress and deflection can be described by Stoney equation for the cantilever with a rectangular cross section [8]. Since the static loading induced deflections are usually in the order of tens to hundreds of one nanometer, detection of such small deflection is a challenge. Dynamic mode of microcantilever has been designed to detect small variations in mass load resulting from adsorption and desorption of molecules [9]. The resonance frequencies of cantilever vary with the additional analyte. Take the recent invented nanocantilever sensors for example, the sensitivity could achievel attogram, i.e., $10^{-18} \mathrm{~g}$.

The sensitivity of micro- and nano-cantilever biosensors depends on the measurement techniques and the cantilever design including material, shape, profile, dimension, etc. For example, a highly flexible cantilever will lead to relatively larger deflection for effective detection. However, this is at the expense of an increase in noise level, which will affect the accuracy of resonant frequency of cantilevers. Thus, optimal design of cantilevers to obtain the high deflection abilities and sufficient frequency characteristics is in immediate need [10]. Ansari et al. [11] recently proposed a stepped cantilever with a $\mathrm{u}$-shaped piezoresistor and enhanced the design through finite element modeling.

Finite element method has been increasingly used to investigate the performance of various biosensor designs by evaluating the influence of cantilever parameters on its static deflection and natural frequency. The deflection induced by the surface stress is generally simulated by action of an equivalent in-plane tensile force on the surface of the cantilever. Being a major source of noise, the thermal drifting should be considered. In addition, the spatial arrangement of the cantilevers array could also be a source of noise. Studies in this aspect remain largely unexplored. Furthermore, biosensors are usually operated in liquid environment. Flow excitation may considerably influence dynamic properties of cantilevers. Fluid damping is speculated to cause the discrepancy between theoretical and experimental dynamic analysis [12]. The computational modeling of microcantilever biosensors operating in liquid environment is also lacking.

The publication of studies concerning simulation and optimization of microcantilever biosensors in an open access journal such as Journal of Biosensors and Bioelectronics is essential to gain insights on the potential of these sensors as well as to advance their design. The computational modeling of coated microcantilever or nanocantilever biosensors have not yet been fully exploited, which may allow the biologist to probe the living cells or molecule within specific physiological or pathological settings. Readers will mostly appreciate the broader applications of simulation and optimization methodology to the area of biomedical or drug discovery.

\section{Reference}

1. Joshi R, Joshi RM (2006) Biosensors. Gyan Publishing House.

2. Wu G, Datar RH, Hansen KM, Thundat T, Cote RJ, et al. (2001) Bioassay of prostate-specific antigen (PSA) using microcantilevers. Nat Biotechnol 19: 856860 .

3. Lee CY, Lee GB (2003) Micromachine-based humidity sensors with integrated temperature sensors for signal drift compensation. J Micromech Microeng 13 620.

4. Raiteri R, Nelles G, Butt HJ, Knoll W, Skladal P (1999) Sensing of Biological Substances Based on the Bending of Microfabricated Cantilevers. Sens Actuators B Chem 61: 213-217.

5. Cherian S, Guptab RK, Mullin BC, Thundat T (2003) Detection of heavy metal ions using protein-functionalized microcantilever sensors. Biosens Bioelectron 19: 411-416.

6. Barnes JR, Stephenson RJ, Welland ME, Gerber Ch, Gimzewski JK (1994) Photothermal spectroscopy with femtojoule sensitivity using a micromechanical device. Nature 372: 79-81.

7. Hwang KS, Lee SM, Kim SK, Lee JH, Kim TS (2009) Micro-and nanocantilever devices and systems for biomolecule detection. Annu Rev Anal Chem (Palo Alto Calif) 2: 77-98.

8. Stoney GG (1909) The tension of metallic films deposited by electrolysis. Proc R Soc Lond 82: 172-175.

9. Garg V (2006) Indirect measurement of complex modulus of thin aluminum nitride piezoelectric film by modal vibrations analysis. ProQuest.

10. Fadel L, Dufour I, Lochon F, Francais O (2004) Signal-to-noise ratio of resonant microcantilever type chemical sensors as a function of resonant frequency and quality factor. Sens Actuators B Chem 102: 73-77.

11. Ansari MZ, Cho C, Urban G (2012) Stepped piezoresistive microcantilever designs for biosensors. J Phys D: Appl Phys 45: 215401.

12. Lévy R, Maaloum M (2002) Measuring the spring constant of atomic force microscope cantilevers: thermal fluctuations and other methods. Nanotechnology 13: 33.

*Corresponding author: Linxia Gu, Department of Mechanical Engineering, University of Nebraska-Lincoln, USA, Tel: 402-472-7680; Fax: 402-472-1465; E-mail: Igu@unl.edu

Received August 27, 2012; Accepted August 29, 2012; Published September 01,2012

Citation: Gu L (2012) Simulation and Optimization of Microcantilever Biosensors. J Biosens Bioelectron 3:e113. doi:10.4172/2155-6210.1000e113

Copyright: (c) $2012 \mathrm{Gu}$ L. This is an open-access article distributed under the terms of the Creative Commons Attribution License, which permits unrestricted use, distribution, and reproduction in any medium, provided the original author and source are credited. 